J. Urol. Urogynäkol. AT 2019 • 26:20-22 https://doi.org/10.1007/s41972-019-0063-5 Online publiziert: 20. Februar 2019 (c) Der/die Autor(en) 2019

\section{Einleitung}

Als Mikrobiom bezeichnen wir das gesamte Genom aller Bakterien, Viren und Pilze in einem Organismus bzw. in einem Organsystem (z. B. im menschlichen Magen-Darm-Trakt). Als Mikrobiota bezeichnen wir die Organismen, also die Bakterien, Viren und Pilze selbst. Mikrobiota können als Symbionten, Kommensalen, Parasiten oder Pathogene in einem Organismus existieren. Symbiose ist das Zusammenleben von Individuen zweier unterschiedlicher Arten, von dem beide Partner profitieren. Kommensalismus ist das Zusammenleben von Individuen verschiedener Arten, welches für Angehörige der einen Art positiv, für diejenigen der anderen Art neutral ist. Parasitismus ist das Zusammenleben von Individuen verschiedener Arten, das für den Parasiten von Vorteil, für den Wirt aber von Nachteil ist. Die Begriffe Mikrobiom und Mikrobiota werden oft synonym verwendet.

\section{Menschliches Mikrobiom}

Schon länger ist die Tatsache bekannt, dass menschlicher Urin auch bei beschwerdefreien Menschen nicht steril ist. Erst allmählich aber entwickelt sich ein Verständnis für die Implikationen dieser Erkenntnis: Zum Beispiel ist ein unsteriler Harn nicht gleichbedeutend mit Infektion oder Erkrankung.

Wie so oft basiert auch dieses neue Wissen auf technischen Fortschritten, in diesem Fall auf verfeinerten Methoden, um Mikroorganismen nachzuweisen.

„Man kann nur finden, wenn man weiß, was man sucht und es auch erken-

\title{
Wolfgang Umek
}

Universitätsklinik für Frauenheilkunde, Medizinische Universität Wien, Wien, Österreich

\section{Das urogenitale Mikrobiom und seine Bedeutung für den weiblichen Harntrakt}

nen kann. " Dieser mikrobiologische Leitsatz wurde durch die Technik der Sequenzierung von genetischem Material verändert. Denn mittels 16S-rRNA-Sequenzierung kann aus Bruchstücken von genetischem Material auf bekannte und bisher unbekannte Organismen geschlossen werden [1]. Die 16S-rRNA-Sequenzierung verwendet das $16 \mathrm{~S}$ Bruchstück der ribosomalen Ribonukleinsäure RNA als Marker im Sequenzierungsprozess. Der Nachteil ist, dass nicht zwischen vitalen Organismen und avitalen Genombruchstücken unterschieden werden kann.

Die 16S-rRNA-Sequenzierung wurde im Rahmen des „Human Microbiome Project“ (HMP) eingesetzt [6]. Dieses US-amerikanische, hochdotierte Forschungsprojekt widmete sich in seiner ersten Phase von 2007-2014 der Kategorisierung des Mikrobioms in den menschlichen Organen Nase, Ohr, Mund, Vagina und Darm. Das Mikrobiom des Harntraktes war nicht Teil des HMP.

Aus dem HMP hervorgegangen ist die Erkenntnis, dass das Verhältnis von Bakterien zu somatischen Zellen im menschlichen Organismus annähernd 1:1 beträgt. Das menschliche Genom enthält ca. 23.000 Gene, das Mikrobiom weitere 3 Mio., die zumindest teilweise dem Wohl des Menschen dienen. So ist z. B. die Tatsache bekannt, dass Darmbakterien bei der Verdauung von Nährstoffen helfen.

Von 100 isolierten Bakterienstämmen sind 4 für den Menschen relevant: $A c$ tinobacteria, Bacteroides, Firmicutes und Proteobacteria. Dieses „core microbiome“ des Menschen verändert sich mit zunehmendem Lebensalter. Es ist aller- dings $z u$ früh, um ein altersspezifisches Mikrobiom zu definieren. Auch konnte bis dato noch kein geschlechtsspezifisches Mikrobiom für die Organsysteme Nase, Ohr, Mund und Darm definiert werden.

\section{Mikrobiom und überaktive Blase}

Über das Mikrobiom der Blase bei gesunden, asymptomatischen Menschen wissen wir, dass es Corynebacterium spp., Streptococcus spp., Actinomyces spp., Staphylococcus spp., Aerococcus spp., Bifidobacterium spp., Ureaplasma spp., Actinobaculum und überraschenderweise Gardnerella enthalten kann. Das Blasenmikrobiom von Frauen ist heterogener und weist eine größere Diversität auf als jenes von Männern.

Mögliche physiologische Funktionen des Blasenmikrobioms sind:

- Erhalt einer protektiven Schicht und Barriere gegen pathogene Keime

- Verdrängungswettbewerb gegen pathogene Keime um Platz am Epithel

- Verhinderung der Adhäsion von pathogenen Keimen am Urothel

- Verdrängungswettbewerb gegen pathogene Keime um Nährstoffe

- Produktion von antimikrobiellen Wirkstoffen durch das Mikrobiom

- Stimulation der wirtseigenen Immunabwehr gegen pathogene Keime

- Rolle bei der Entstehung eines intakten Urothels (Actinobaculum, Actinomyces, Aerococcus, Arthrobacter, Corynebacterium, Gardnerella, 
Oligella, Staphylococcus und Streptococcus)

Es gibt bereits Ergebnisse aus Studien, die einen Zusammenhang zwischen der Zusammensetzung des Mikrobioms und dem Syndrom der „überaktiven Blase“ („overactive bladder“ $[\mathrm{OAB}]$ ) untersuchten. Interessant ist dabei zunächst, dass die Diagnose dieses Syndroms bisher unbedingt den Ausschluss einer entzündlichen Komponente verlangte - entsprechend einer traditionellen Überzeugung, wie sie exemplarisch aus einem englischen Zitat hervorgeht: „... these terms can be used if there is no proven infection or other proven pathology“ [2]. Im aktuellen Kontrast zu dieser möglicherweise bald historischen Lehrmeinung wurde in einer Studie gezeigt, dass im Harn eines Kollektivs von 68 Patientinnen mit $\mathrm{OAB}$ die Keimarten Actinobaculum, Actinomyces, Aerococcus, Arthrobacter, Corynebacterium, Gardnerella, Oligella, Staphylococcus und Streptococcus signifikant häufiger vorkommen als in einem Kollektiv von 50 gesunden Frauen [3]. Umgekehrt ist Lactobacillus crispatus häufiger bei gesunden Frauen nachweisbar als bei solchen, die an OAB erkrankt sind.

Unterschiede im Mikrobiom finden sich interessanterweise auch bei OABPatientinnen, die unterschiedlich gut auf den anticholinergen Wirkstoff Solifenacin ansprechen [4]. Harn von sog. Respondern (OAB-Patientinnen, deren Symptome sich auf Solifenacin besserten) enthielt weniger Bakterien und eine geringere Diversität vor Beginn der Behandlung, Harn von sog. Nonrespondern hingegen eine höhe Diversität an Bakterien und oft untypische Bakterienstämme.

Eine erste kontrollierte randomisierte Interventionsstudie mit Plazebo [5] untersuchte die Wirkung von Lactobacillus crispatus, wenn intravaginal appliziert: Die Rate von rezidivierenden Harnwegsinfekten betrug in der Interventionsgruppe $15 \%$ - vs. $27 \%$ in der Kontrollgruppe (RR: $0,5, p<0,1$ ).

\section{Schlussfolgerung}

Die überaktive Blase war bisher ein ebenso rätselhaftes wie quälendes Leiden, das

J. Urol. Urogynäkol. AT 2019 ·26:20-22 https://doi.org/10.1007/s41972-019-0063-5

(c) Der/die Autor(en) 2019

\section{W. Umek \\ Das urogenitale Mikrobiom und seine Bedeutung für den weiblichen Harntrakt}

\section{Zusammenfassung}

Entgegen der landläufigen Annahme einer "Sterilen Blase" weist die gesunde weibliche Harnblase ein vielfältiges Mikrobiom auf. Die Erforschung der Zusammensetzung und Bedeutung der darin enthaltenen Bakterienstämme steht aber erst am Anfang. Es gibt erste schlüssige Hinweise für signifikante Unterschiede zwischen den Mikrobiomen von gesunden Frauen und Patientinnen mit Symptomen des unteren Harntraktes. Damit eröffnet sich auch ein weites Forschungsgebiet für den Einsatz von Probiotika zur Behandlung von Blasenfunktionsstörungen.

Schlüsselwörter

Mikrobiom $\cdot$ Harnblase $\cdot$ Blasenfunktionsstörung · Probiotika $\cdot$ Überaktive Blase

\section{The urogenital microbiome and its relevance to the female urinary tract}

\section{Abstract}

Contrary to the common scientific belief of a "sterile bladder", the healthy female bladder has a diverse microbiome. Research into understanding the diversity and importance of specific bacterial species to the healthy female bladder microbiome is in its infancy. Results of scientific studies suggest significant differences between the microbiome of patients with lower urinary tract symptoms compared to healthy controls. This opens a wide field of research into the use of probiotics to treat bladder function disorders.

\section{Keywords}

Microbiome - Urinary bladder - Bladder function disorder · Probiotics - Overactivebladder syndrome vorzugsweise das weibliche Geschlecht betrifft. Fortschritte in der Erforschung des menschlichen Mikrobioms konnten zeigen, dass der keineswegs sterile Harn des gesunden Menschen sich in der bakteriellen Zusammensetzung von dem der von einer OAB Betroffenen unterscheidet. Damit erschließen sich neue Perspektiven für die Untermauerung der Diagnose sowie auch für wirksame Therapien mit Probiotika.

\section{Korrespondenzadresse}

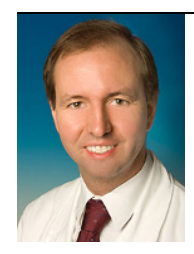

Ao. Univ.-Prof. Dr. Wolfgang Umek Universitätsklinik für Frauenheilkunde, Medizinische Universität Wien Währinger Gürtel 18 1090 Wien, Österreich wolfgang.umek@ meduniwien.ac.at
Funding. Open access funding provided by Medical University of Vienna.

\section{Einhaltung ethischer Richtlinien}

Interessenkonflikt. W. Umek gibt an, dass kein Interessenkonflikt besteht.

Dieser Beitrag beinhaltet keine vom Autor durchgeführten Studien an Menschen oder Tieren.

Open Access. Dieser Artikel wird unter der Creative Commons Namensnennung 4.0 International Lizenz (http://creativecommons.org/licenses/by/4.0/deed de) veröffentlicht, welche die Nutzung, Vervielfältigung, Bearbeitung, Verbreitung und Wiedergabe in jeglichem Medium und Format erlaubt, sofern Sie den/die ursprünglichen Autor(en) und die Quelle ordnungsgemäßnennen, einen Linkzur Creative Commons Lizenz beifügen und angeben, ob Änderungen vorgenommen wurden.

Hinweis des Verlags. Der Verlag bleibt in Hinblick auf geografische Zuordnungen und Gebietsbezeichnungen in veröffentlichten Karten und Institutsadressen neutral. 


\section{Literatur}

1. Fukuda K, Ogawa M, Taniguchi H, Saito M (2016) Molecular approaches to studying microbial communities: targeting the $16 \mathrm{~S}$ ribosomal RNA gene. JUOEH 38(3):223-232

2. Abrams P et al (2003) The standardisation of terminology in lower urinary tract function: report from the standardisation sub-committee of the International Continence Society. Urology 61(1):37-49

3. Pearce et al (2014) The female urinary microbiome: a comparison of women with and without urgency urinary incontinence. MBio. https://doi.org/10. 1128/mbio.01283-14

4. Thomas-White KJ et al (2016) Incontinence medication response relates to the female urinary microbiota. Int Urogynecol J 27(5):723-733

5. Stapleton AE et al (2011) Randomized, placebocontrolled phase 2 trial of a lactobacillus crispatus probiotic given intravaginally for prevention of recurrent urinary tract infection. Clin Infect Dis 52(10):1212-1217

6. The Human Microbiome Project Consortium (2012) A framework for human microbiome research. Nature 486:215-221

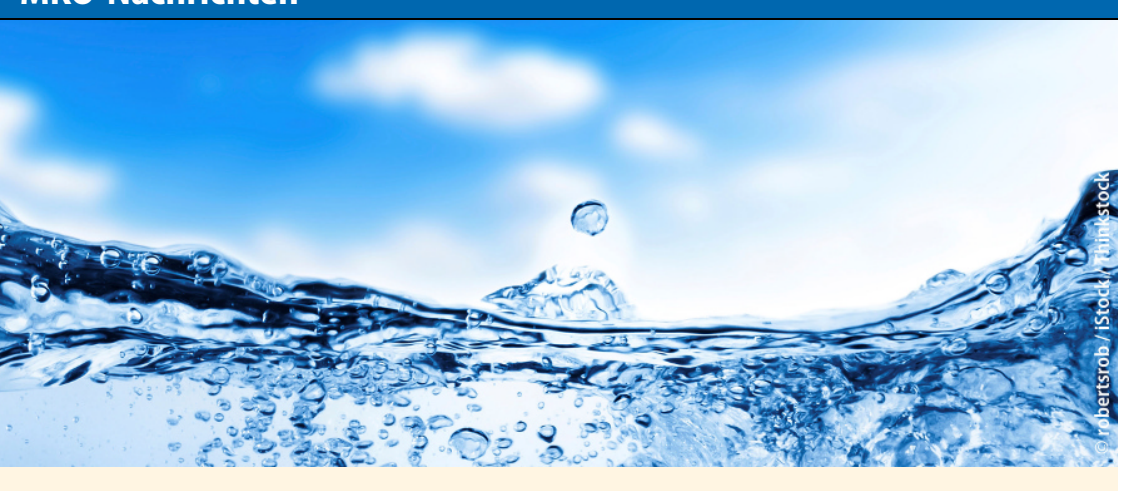

MKÖ-Veranstaltungen 2019

\section{Jahrestagung der Medizinischen Kontinenzgesellschaft Österreich (MKÖ)}

18.-19. Oktober 2019

LFI Oberösterreich

Seminarhaus auf der Gugl

Auf der Gugl 3, 4021 Linz

\section{Welt-Kontinenz-Woche}

17.-23. Juni 2019

Die Aktionswoche wird von der ICS (International Continence Society) ausgerufen und der World Federation of Incontinence Patients (WFIP) organisiert. Die MKÖ nimmt dies zum Anlass, um in Österreich Aktivitäten zu setzen, das Thema Inkontinenz in den Fokus zu rücken und die Öffentlichkeit / Betroffene über Inkontinenz (Formen, Häufigkeit und Behandlungsmöglichkeiten) zu erreichen, zu sensibilisieren, zu informieren und schließlich zu mobilisieren.

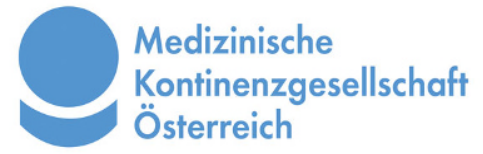

\section{Regionale Fortbildungen}

Der "Kontinenz-Stammtisch“ in Oberösterreich, Wien und Salzburg bzw. das „Kontinenz-Meeting "in Kärnten sind Fortbildungsund Vernetzungs-Veranstaltungen der MKÖLandesstellen und richten sich an Fachleute aus den jeweiligen Bundesländern, die sich mit Diagnostik und Therapie von Inkontinenz beschäftigen. Betreuer/innen von Menschen mit Harn-und Stuhlinkontinenz oder Entleerungsstörungen aus dem ärztlichen, pflegenden und physiotherapeutischen Bereich tauschen sich in angenehmer Atmosphäre aus und intensivieren ihre Zusammenarbeit. Im Rahmen der Veranstaltungen wird ein Experten-Netzwerk auf- bzw. ausgebaut. Damit kann die Betreuung der Betroffenen flächendeckend und wohnortnahe durchgeführt werden.

Die nächsten Termine (mehr Info jeweils vor den Veranstaltungen auf www.kontinenzgesellchaft.at):

\section{- 7. Wiener Kontinenz-Stammtisch}

4. April 2019

Akademie für Fortbildungen und

Sonderausbildungen

Spitalgasse 23, 1090 Wien

\section{- 15. OÖ Kontinenz-Stammtisch}

21. Mai 2019

Med Campus III., Kepler Universitätsklinikum Krankenhausstraße 9, 4021 Linz

\section{- Kontinenz Fachmeeting Kärnten}

27. Juni 2019

Hotel Sandwirth

9020 Klagenfurt 